\title{
How significant is erosion in extrusion- insights from analogue and analytical models
}

\author{
Soumyajit Mukherjee ${ }^{*}$, Hemin A Koyi², Christopher J Talbot² and AK Jain ${ }^{3}$ \\ 1 Engineering Mechanics Unit, Jawaharlal Nehru Centre for Advanced Scientific Research, Jakkur, Bangalore-560064, INDIA \\ 2 Hans Ramberg Tectonic Laboratory, Uppsala University, 75236 Uppsala, SWEDEN \\ 3 Department of Earth Sciences, Indian Institute of Technology Roorkee, INDIA \\ * For correspondence, email: soumyajitm@gmail.com
}

The absolute role of climate/erosion in extrusion of mountains has been debated for a long time. Field-studies, micro-structural observations and few analytical trials confirm that the extrusion of the Higher Himalayan Shear Zone, Sutlej section took place initially by simple shearing and was followed by combined simple shear and channel flow in a shifting mode (Mukherjee 2007). The extrusion mechanism of the HHSZ is studied with 10 analogue models of channel flow initiating from a horizontal channel and extrusion through a linked inclined channel. The inclined channel is the model HHSZ and is of parallel, gently diverging-up and strongly diverging-up geometries in different considerations. In these experiments, Polydimethylsiloxane (PDMS), a transparent Newtonian viscous polymer is used as the model material and geometric- and dynamic similarities are maintained with the prototype. Six flow zones are deciphered in the two channels in the mature stage of extrusion of the PDMS. Parabolic profiles are produced at the middle of both the inclined- and the horizontal channel during a Poiseuille and Jeffery Hamel flows. The part of the PDMS originally inside the horizontal channel starts moving through the inclined channel at a faster rate than the part of the PDMS initially residing in the inclined channel. This in effect gives rise to thrust movement of the former part of the PDMS. The thrust plane originates at the corner joining the inclined and the horizontal channel and rotates while coming closer to the free surface. The tectonic insights gained from these experiments are (i) secondary ductile thrusting took place in the HHSZ as a delayed response to its channel flow mode of extrusion, (ii) the genesis of the thrust seems to be related to the change in the direction of extrusive flow- from horizontal to inclined up; (iii) as these models were performed without any erosion of the extruded PDMS, erosion induced by climate did not trigger nor was a deciding factor in the genesis of the secondary thrust and concomitant extrusion of the HHSZ. However, erosion induced by a disparity in precipitation on the HHSZ might have augmented the extrusion process i.e. it had a passive role. In Sutlej section of the HHSZ, the simulated thrust can be correlated with the Chaura Thrust with the recorded activation at least $13 \mathrm{Ma}$ after the ongoing extrusion of the HHSZ by channel flow mechanism around $18 \mathrm{Ma}$.
Salt diapirs are spectacular natural structures and are products of an interaction between the extrusive force that build and erosion that tends to degrade them. While in humid climate, their erosion rate can reach few $\mathrm{mm}$ per year, in arid climates it goes down to one tenth to that of the former rate (Bruthans et al. 2008). Salt diapirism has been modeled as a product of the density difference between the salt and the surrounding limestone country rock in the islands of Hormuz and Namakdan in the Persian Gulf (Mukherjee et al. 2007). Most of the 200 or so diapirs of Hormuz salt in the Zagros mountains of Iran extrude majestic mountains of salt that rise $400 \mathrm{~m}$ above their strong vents in limestones. Even if a difference in density between the salt and the country rocks are in the order of $10^{-1} \mathrm{gm} . \mathrm{cm}^{-3}$, such a low magnitude persistent over tens of thousands of years can give rise to extrusion of the salt for kilometers that can have dynamic viscosity as high as $1021 \mathrm{~Pa} \mathrm{~s}$ (Mukherjee et al. 2007). Without taking into account their erosion rates, diapirism was modeled as being triggered and maintained by the density contrast. This indicates that erosion is not a crucial controlling factor for the extrusion. Erosion is one out of many components that can, however, modify the pressure gradient controlling the extrusion process. The other components are (i) the up-building extruded mass of salt that imparts temporally increasing weight downwards, and (ii) gravitational spreading that allows the extruded salt to flow away (Weinberger et al. 2006).

\section{References}

Bruthans J, N Asadi, M Filippi, Z Vilhelm. and M Zare. 2008. A study of erosion rates on salt diapir surfaces in the Zagros Mountains, SE Iran. Environmental Geology 53: DOI 10.1007/s00254-007-0734-6

Mukherjee S. 2007. Geodynamics, Deformation and Mathematical Analysis of Metamorphic Belts, NW Himalaya. Unpublished Ph.D. thesis. Indian Institute of Technology Roorkee: $267 \mathrm{p}$

Mukherjee S, CJ Talbot, HA Koyi. 2007. Estimation of viscosity of natural salts of the Hormuz and Namakdan salt diapirs in the Persian Gulf. Annual Transaction of Nordic Rheology Society 15: 189-196

Weinberger R, V Lyakhovsky, G Baer and ZB Begin. 2006. Mechanical modeling and InSAR measurements of Mount Sedom uplift, Dead Sea basin: Implications for effective viscosity of rock salt. Geochemistry Geophysics and Geosystems 14: DOI: 10.1029/2005GC001185 